TM-1543

\title{
Time Variations of Fields in Superconducting Magnets and Their Effects on Accelerators*
}

D. A. Herrup, M. J. Syphers, D. E. Johnson, R. P. Johnson,

A. V. Tollestrup, R. W. Hanft, B. C. Brown, M. J. Lamm, M. Kuchnir, and A. D. McInturff

Fermi National Accelerator Laboratory

P.O. Box 500, Batavia, Illinois

August 22, 1988

*Presented at the 1988 Applied Superconductivity Conference, San Francisco, California, August 21-25, 1988. 
D. A. Herrup, M. J. Syphers, D. E. Johnson, R. P. Johnson, A. V. Tollestrup, R. W. Hanft, B. C. Brown, M. J. Lamm, M. Kuchnir, A. D. McInturff

Fermi National Accelerator Laboratory* P. O. Box 500

Batavia, Illinois 60510

\section{Abstract}

A report on the time dependence of magnetic fields in the superconducting magnets of the Fermilab Tevatron has been published. A field variation of order 1 Gauss at the aperture radius is observed. Studies on both full sized Tevatron dipoles and prototype magnets have been used to elucidate these effects. Explanations based on eddy currents in the coil matrix or on flux creep in the superconducting filaments are explored with these tests. Measurement results and techniques for controlling the effect based on new laboratory tests and the latest accelerator operation are presented.

\section{Introduction}

Successful operation of the Tevatron accelerator at Fermilab has demonstrated the need for a deeper understanding of the magnetic fields produced in superconducting magnets. In previous accelerators it was necessary to understand the magnetic field dependence on excitation current, on excitation rate (eddy current effects) and on hysteresis effects (due to hysteresis in iron). In superconducting magnets it was known that hysteresis due to diamagnetic effects in the superconducting materials would create important hysteretic field changes. Measurements of the chromaticity of the beam in the Tevatron revealed that important changes of the sextupole fields existed over times ranging from seconds to hours'. This has stimulated detailed studies of the materials ${ }^{2}$ and magnets $^{3}$ which are used in the Tevatron in an attempt to further understand these effects. Based on these results, new operational schemes have been utilized to control the chromaticity of the accelerator both during the injection time and during initial stages of acceleration. This paper will summarize our present status in efforts to understand these effects and present new measurements of accelerator operating parameters which indicate the success and limitations of our undertaking.

\section{Creating Magnetic Fields}

To study the magnetic fields of superconducting magnets it is useful to describe the sources in a general calculational framework. We write

$$
\mathrm{B}=\mathrm{B}_{\mathrm{tc}}+\mathrm{B}_{\mathrm{fei}}+\mathrm{B}_{\mathrm{mag}}+\mathrm{B}_{\mathrm{fes}}+\mathrm{B}_{\mathrm{edd}}
$$

where $B_{t c}$ is the transport current contribution, $B_{f e i}$ is the field ${ }^{\text {tc }}$ enhancement due to infinite permeability fei iron, $B_{\text {mag }}$ is due to magnetization effects in the superconductor and the iron, $B_{\text {feg }}$ is the correction for iron saturation, and $B_{\text {eddy }}$ is the contribution due to eddy currents. For (conductor dominated) superconducting synchrotron magnets, $B_{t c}$ is designed to

*Work supported by the U. S. Department of Energy

Manuscript received August 22, 1988. provide the desired field shape and strength with enhancement from $B_{\text {fei }}$. The other terms can create error fields whose magnitude must be controlled to provide a suitable field quality to match the requirements of the accelerator. High energy accelerators such as the Fermilab Tevatron require field uniformitics approaching $10^{-4}$ over a range from particle injection field of about $.66 \mathrm{~T}$ to maximum particle energy at or above $4 \mathrm{~T} \quad(1 \mathrm{TeV}=4.4 \mathrm{~T})$. This framework provides a suitable mechanism for calculating the largest corrections, however, there is no rigorous basis for the separation of these effect and there may be interactions between the separate terms.

Studies of the field errors under conditions expected for rapid cycling operation " revealed fields of several Gauss at the aperture radius of $2.54 \mathrm{~cm}$ due to magnetization effects in the superconducting cable. These error fields have a rich harmonic structure with important terms corresponding to the "allowed" symmetries for the magnets (6-pole and 10-pole and higher for dipole magnets, 12-pole and higher for quadrupoles). Following observations of beam effects which suggested time dependences of these ficlds, 1 extensive studies of these fields have been carried out.

In attempting to understand the sources of these time varying fields we have examined two classes of models. The "natural" time constant of copper $(\mu \sigma)$ at low temperatures suggests that eddy current fields in cryogenic magnets may have time constants which are much longer than the times associated with eddy currents in magnets with room temperature copper coils. Eddy current time constants are set by the arca aud resistance of coupling loops. Since the multi-filamentary superconducting structure interconnects with the copper in a complex fashion, we have only deduced very general properties of these solutions at this point. An alternative mechanism involves the time dependent magnetization effects in the superconducting matrix. Here it is known that flux quanta must penetrate the (Type II) superconducting filaments in order to support the very high magnetic fields of these magnets. Flux motion in superconductors has been studied in a variety of systems, but the available information is, again, not sufficient to provide a complete calculational model. Instead we will outline the elements of these contrasting models and attempt to relate the magnitude and time dependence of the observed effects to known features of the models.

\section{Properties of eddy current models}

An eddy current source term can be electrically modeled using a transmission line. This results in the observation that the characteristic time variation is exponential. The time constant $\tau$ of loops depend on $\mathrm{L}^{2} \mu \sigma$ where $\mathrm{L}$ is the length of the loop (in meters), $\mu$ is the permeability and $\sigma$ is the conductivity. In copper at liquid helium temperatures, a $1 \mathrm{~cm}$ loop has a time constant of about 0.75 seconds. In a complicated structure such as a multifilament magnet, we might find many different time constants. To examine our data for 
evidence of these effects we will discuss two limiting cases: long time constants and short time constants. The characteristic time of our excitation is the ramp-up time of the magnets. For Tevatron magnets this is 20 seconds.

Long time constant effects will be revenled by slow tails (sum of exponentials) and an overall dependence of the $b_{2}$ on ramp history but only weakly on ramp rate. Short time constant effects will cause changes which depend on ramp rate but not multi-ramp history. Eddy current effects will not depend on absolute field level and will be symmetric between increasing and decreasing currents.

\section{Persistent Current Magnetization Effects}

An effective description of the magnetization created by persistent current effects in multifilamentary cables has been created for the quasistatic case, ${ }^{4,5}$ This model relies on describing the current distribution within the filaments and results in calculations which describe the magnetic fields in terms of the critical current, $J$, the filament diameter, $d$, and a parameter, $\epsilon$, which describes the penetration of the filaments. An equivalent description which can be more easily tied to measurable properties of the materials can be formulated which utilizes the magnetization vs applied field for the superconducting cable. ${ }^{6}$ Examination of these models will show that the magnetization distribution which establishes the various harmonic field components is determined by the local (dipole) field magnitude and direction and its history. This suggests that harmonic fields due to these effects will be closely related under various conditions.

The Meissner effect which creates the shielding currents in these materials is not complete in Type II superconductors. The flux penetration which allows high field superconductivity requires the penetration of quantized flux vortices through the superconducting materials. Studies of the motions of these flux vortices have been a part of the effort to understand high field superconducting materials. Their effects on magnetic fields of superconducting accelerator magnets has not been discussed, however. Pinning of these vortices at imperfections in the metal lattice allow the creation of large magnetic fields. Motion of the vortices under thermal excitation has been shown to result in field changes characterized by ( $\ln t)$ time dependences. Such effects are apparent in our magnets.

A $6 \mathrm{~m}$ long Tevatron Dipole when operated at $2 \mathrm{~T}$ (about $2000 \mathrm{~A}$ ) will contain about $2 \mathrm{~T}-\mathrm{m}^{2}$ of magnetic flux. A quantum of flux is about $2 \times 10-^{15}$ $T-m^{2}$. Thus about $10^{15}$ flux quanta will be pinned in a Tevatron magnet. There are 2100 filament of 9 micron diameter in a strand and 23 strands per cable for the Tevatron dipoles. Each quadrant of the coil uses 56 turns so there are about $10^{7}$ filaments $6 \mathrm{~m}$ long. We sce then that for each 10 micron $\left(10^{-5} \mathrm{~m}\right)$ length of cable we have of order $10^{2}$ flux quanta. Motions of these flux quanta are governed by both the transport current and shielding currents in the filaments.

Studies of changing magnetic fields due to flux vortex motion in Type II superconductors ("flux creep") have been carried out for more than 25 years? ${ }^{7}$. The dominant physical process was explained by Anderson ${ }^{8}$ in 1962 as the thermally activated motion of flux quanta through the array of pinning sites. Studies with various types of materials have confirmed some aspects of this theory but a number of points remain unclear. Beasley et. $a l^{7}$ have demonstrated a number of important effects including changes in the rate of flux motion when the time variations of the flux have not left the materials in the "critical state", dependence of the creep rate of magnetic field level and the existence of discrete event in which the motions involve from 1 to $10^{2}$ events. Studies involving NbTi multifilamentary wires have been carried out by Chaussy et. al. but under conditions very different than those in our magnets. Recrntly, some of us have begun studies ${ }^{2}$ of the materials characteristic of modern high field superconducting magnets. Nonetheless, neither theory nor experiment is yet able to provide a complete basis for explaining the time dependence of the fields in our magnets.

\section{b2 Time Development in RL1001}

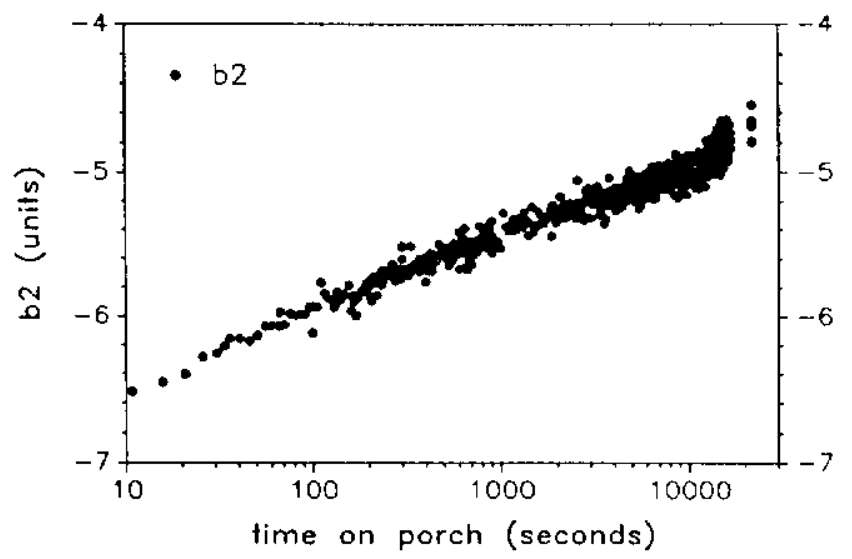

Figure 1. Time dependence of sextupole harmonic for $0.8 \mathrm{~m}$ Tevatron dipole model. Data taken at $800 \mathrm{~A}$ after standard accelerator ramp. Data is well represented by $b_{2}=-7+.217(\ln t)$.

\section{Analysis of Laboratory Measurements}

Hanft et. al. ${ }^{3}$ have reported on some of the measurements we have taken in an attempt to understand these time dependent fields. In addition we performed a series of tests to look specifically for long time constant eddy current effects. Beginning after a magnet quench, we observed $b_{2}$ on successive ramps until it stopped changing. After a few ramps (hundreds of seconds) the field was the same on successive ramps within our ability to measure. On the other hand, fichd decay is still measurable after thousands of seconds. We also compared the change in $b_{2}$ at $800 \mathrm{~A}$ after two different ramping conditions. In the first we ramped from 0 to 800 and observed while remaining at $800 \mathrm{~A}$ that $b_{2}$ becomes less negative (smaller absolute value). We compare this result to one in which we ramped from 0 to 800 , then 800 to 900 to $800 \mathrm{~A}$ (at the same ramping rate). A long time constant eddy current model would predict that the effects of the voltage which changed the current between 800 and $900 \mathrm{~A}$ would almost cancel, and in any case the net effect would result in $\mathrm{db}_{2} / \mathrm{dt}$ of the same sign as before. This ramp to $900 \mathrm{~A}$ and back is sufficient to nearly reverse the magnetization ${ }^{4}$. Our measurements show in this case that $\mathrm{db}_{2} / \mathrm{dt}$ is reversed, suggesting that the time varying fields are a magnetization effect.

To illustrate the time dependance we display $\mathrm{b}_{2}$ vs time on the injection porch for the model magnet studied by Hanft et. al. ${ }^{3}$ in Figure 1 . The (ln t) dependence which is characteristic of "flux creep" is evident. The logarithmic decay rate is a useful way to parameterize these results. For normalization, we have previously shown that the magnetization effects observed with increasing magnetic field are approximately the same magnitude and opposite in sign to those observed 
with decreasing field. This leads us to identify the magnitude of the superconducting magnetization sextupole with one half of the difference between the values observed at the same current on increasing and decreasing ramps. This gives 6 units of $b_{2}$ or $B_{2}$ of 4 Gauss at $2.5 \mathrm{~cm}$. Given a logarithmic decay rate of 0.5 units per decade of time we conclude that $1 / b_{2}$ $\mathrm{db}_{2} / \mathrm{d}(\ln \mathrm{t})=.036$ at a field of $0.6 \mathrm{~T}$. $\left\langle b_{2}\right\rangle$ Measurements from Tev

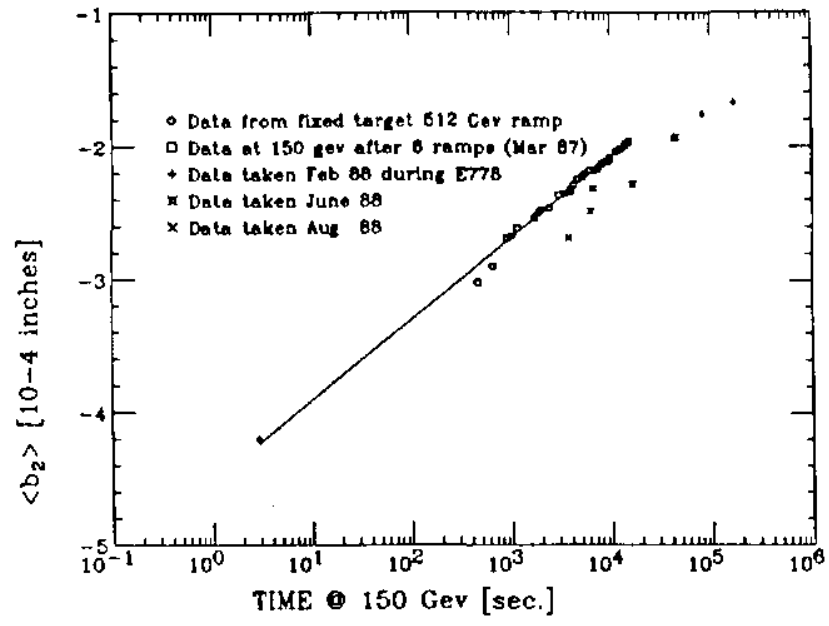

Figure 2. Average sextupole harmonic of dipoles in FNAL Tevatron as determined by transuerse beam motion and sextupole correction strength. Data from 1987 is well represented by $b_{2}=-4.518+.263(\ln t)$

\section{Accelerator measurements}

Finley et. al. ${ }^{1}$ have previously reported measurements from early 1987 of tune and chromaticity of the Tevatron which were time dependant at constant current. In Figure 2 we show extensions of these measurements. Based on the $(\ln t)$ dependence observed in laboratory measurements, we now plot the accelerator based measurements in the same format. For all cases, the sextupole value was inferred from the machine chromaticity. The transverse tune vs radius was utilized to measure the chromaticity at very short times. The remaining data is obtained from measurements of the correction which must be applied to achieve zero chromaticity. This condition is signaled by the observation of a "head-tail" instability which is observed in the transverse beam motion. The 1987 and 1988 data comprise distinct sets of results. The change is not understood at this time. However, both data sets are consistent with a time variation which is dominated by ( $\ln t)$ changes. The agreement between the ensemble of magnets as measured by the beam and the single (model) magnet result is satisfactory.

\section{Correction Efforts in the Tevatron}

Based on the above measurements of chromaticity, corrections have been programmed into the sextupole correction system to maintain a small positive chromaticity during the injection period. The corrections for the change in chromaticity during the initial acceleration ramp have been even more important. Prior to the studies reported in $H$ anft et. al. ${ }^{3}$, the sextupole correction was maintained at a constant value (the value to which it drifted by the $(\log t)$ effect) until the ramp correction required a more positive value. When measurements demonstrated that the sextupole approached the value which the ramp without pause would have given, after changes of less than $30 \mathrm{~A}$, a modified correction scheme was selected. In Figure 3 we show the data from the model magnet ${ }^{3}$ in which we plot on the vertical axis the difference between the sextupole which is observed at a given current and that measured on a ramp in which a long pause at injection current has taken place. On the horizontal axis we plot the logarithm of the time since ramping restarted after the $150 \mathrm{GeV}$ pause.

Time Development of Sextupole after Porch

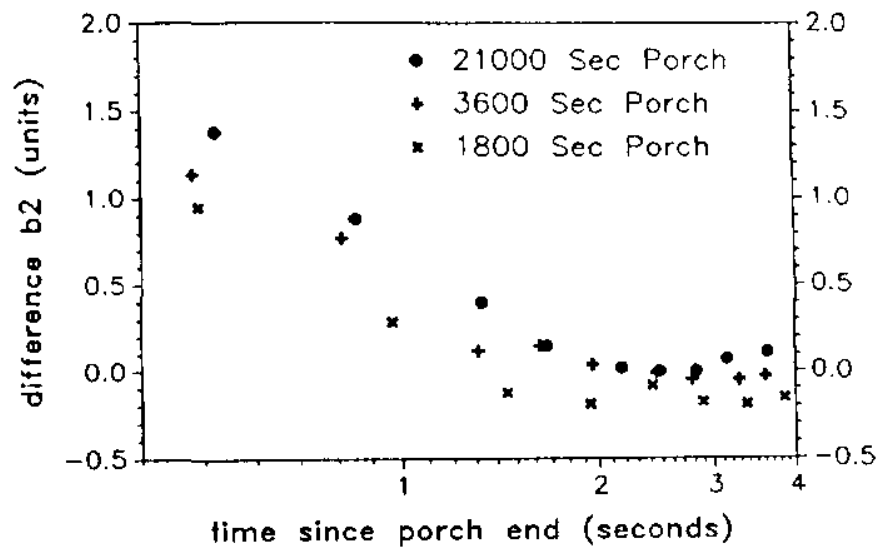

Figure 3. Sextupole harmonic time dependence during initial beam acceleration. The difference between the sextupole observed after long injection porch and sextupole on uninterrupted ramp is plotted.

\section{Normal Sextupole While Pulsing}

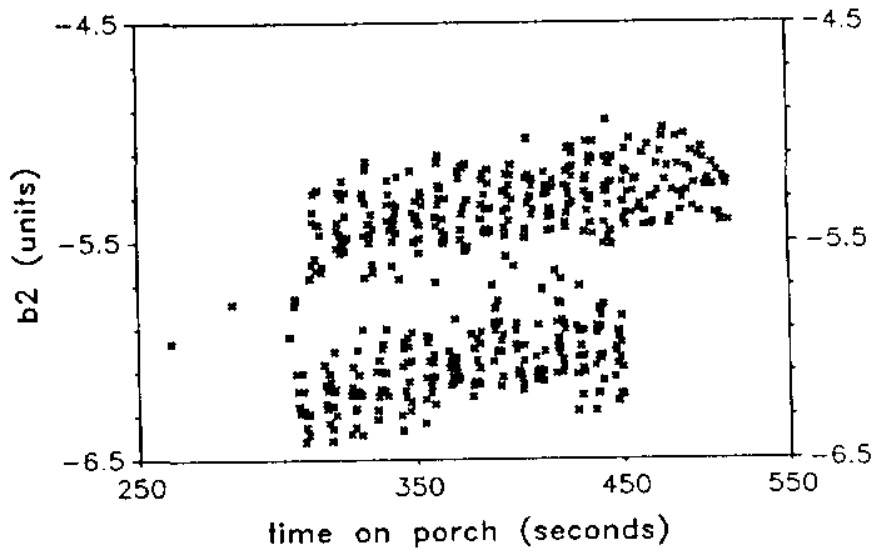

Figure 4. Sextupole harmonic response of a model magnet to a $20 \mathrm{~A}(.1 \mathrm{~Hz})$ square wave modulation of the $800 \mathrm{~A}$ porch current for model magnet RL1001. The two bands of data correspond to the resulting two current levels. The overall drift of the sextupole is not prevented with this excitation.

A correction for the sextupole changes as the ramp begins have been achieved for current Tevatron ramping with a look-up table in which time at injection current and accelerator energy specify a ramping condition. Implementing this correction has had important benefits for operation. The transverse oscillation frequency (tune) of the stored beams is monitored with a frequency analyzer attached to transverse beam monitors. Under normal operation, sharp tune frequency lines are observed. Prior to implementing this correction to the sextupole during the initial ramping, the tune lines became unobservably wide due to the differences in tune for various particle momenta in the beam. With this correction, the tune lines can again be monitored continuously during the acceleration process. This demonstrates the effectiveness of the correction. Phase space dilution of the beam is avoided and the incidence of beam loss during this time in the accelerator cycle is reduced. 
In a search for a technique to reduce the changes in sextupole field during the injection period, a study was made of the response of the field to a square wave modulation of the current near the injection current. A mechanism was sought which would cause the flux pattern to forget previous history. In Figure 4 we have plotted the sextupole field of a model magnet ${ }^{3}$ while a 20 A square wave was added to the 800 A porch current. We observe that the resulting $b_{2}$ is modulated by the current but that the overall (ln t) drift continues. Amplitudes from 1 to $20 \mathrm{~A}$ and frequencies from 0.1 to $10 \mathrm{~Hz}$ were examined without finding evidence that this technique would reduce the sextupole field changes.

\section{Conclusions}

Detailed studies of the time dependance of magnetic fields in superconducting dipoles have revealed:

1. Small but important changes in the fields occur with (ln t) time dependences.

2. Additional effects occur with time scales in the 1-10 second range and amplitudes which are also important for accelerator operation. These effects are modified by the current ramp rate and maximum field achieved on both the current and on previous ramp cycles.

3. In both laboratory and accelerator measurements, some events which might be associated with nonsmooth behavior ("sudden" changes on the relevant time scale) and with deviations at long times from observed ( $\ln t)$ behavior have been seen. Further studies will be required to determine if these are instrumental effects or additional complications of the magnet properties.

Measurements of magnetic fields have been carried out which allow important new corrections to be applied to the accelerator operating cycle. Although theoretical understanding of the processes in terms of the "critical state model" and "flux creep" have provided useful guidelines for plotting the results of these measurements, we have not yet been able to understand the details of our measurements based on these pictures. Long time constant eddy current effects are not sufficient to explain these field changes.

Although we have obtained a sufficient empirical picture from which to apply corrections to the existing Tevatron operation, we observe a large number of as yet puzzling effects which we have not fitted into a coherent phenomenological framework.

The accelerator effects which lead to these magnetic field studies are already important for operation of the Tevatron. It is believed that the larger accelerator systems which are under current consideration (SSC, LHC) will require yet more uniform fields. To achieve these field uniformities will almost certainly require further understanding of these phenomena. If active correction systems are employed then the required information about field variation with ramping conditions will need to be known in detail. We note that if these effects are all associated with magnetization effects in the superconductor, then, perhaps the passive correction 5 chemes based on the utilization of different superconducting materials in suitable angular intervals may result in a decreased sensitivity to these effects. ${ }^{9}$

\section{Acknowledgements}

We would like to thank the personnel in the accelerator operations and magnet testing groups at Fermilab who have made this work possible.

\section{References}

[1] D. A. Finley, D. A. Edwards, R. W. Hanft, R. Johnson, A. D. McInturff, and J. Strait, "Time Dependent Chromaticity Changes In the Tevatron," Proceedings of the 1987 IEEE Particle Accelerator Conference, Washington, D.C., March 16-19, 1987, pp. 151-3. Also Fermilab publication FN-451, March 1987.

[2] M. Kuchnir and A. V. Tollestrup, "Flux Creep In a Tevatron Cable", Р яper LM-5 presented at this conference.

[3] R. W. Hanft, B. C. Brown, D. A. Herrup, M. J. Lamm, A. D. McInturff, M. J. Syphers, "Studies of Time Dependance of Fiells in Tevatron Superconducting Dipole Magnets", Paper LH-4 presented at this conference.

[4] B. C. Brown, H. E. Fisk, and R. Hanft, "Persistent Current Fields in Fermilab Tevatron Magnets", IEEE Trans. on Magnetics 21, No. 2, pp. 979-982, March 1985.

[5] M. A. Green, "Residual Fields in Superconducting Magnets", IEEE Trans. on Nucl. Sci. NS-18, No. 3, pp. 664-664 (1971); M. A. Green, "Residual Fields in Superconducting Magnets", Proc. of the Magnet Technology Conference MT-4, pp. 339 (1972).

[6] H. E. Fisk, R. Hanft, M. Kuchnir, and A. D McInturff "Passive Correction of Persistent Current Multipoles in Superconducting Accelerator Dipoles", Proceedings of the ICFA Workshop on Superconducting Magnets and Cryogenics, Brookhaven National Laboratory, May 12-16,1986, pp. 222.

[7] Y. B. Kim, C. F. Hempstead and A. R. Strand "Critical Persistent Currents in Hard Superconductors", Physical Review Letters 9,306 (1962). M. R. Beasley, R. Labusch and W. W Webb, "Flux creep in type-Il superconductors," Physical Review vol. 181 , pp. 682-700, May 1969. J. Chaussy, J. L. Genicon, J. Mazuer, M. Renard, and A. Sulpice, "Flux Creep Phenomenon in Multifilamentary Superconducting Wires", Phys. Letters $87 \AA, 61$ (1981).

[8] P. W. Anderson, "Theory of Flux Creep in Hard Superconductors" Physical Review Letters 9,309 (1962).

[9] B. C. Brown and H. E. Fisk, "A Technique to Minimize Persistent Current Multipoles in Superconducting Accelerator Magnets', Proceedings of the 1984 Summer Study on the Design and Utilization of the Superconducting Super Collider, Snowmass, Colorado, ed by R. Donaldson and J. G. Morfin, p 336-339. 